\title{
What is the role of consolidative thoracic radiotherapy in the era of chemo-immunotherapy for extensive stage small cell lung cancer?
}

\author{
Kristin A. Higgins ${ }^{1,2}$, Ben J. Slotman ${ }^{1,2}$ \\ ${ }^{1}$ Department of Radiation Oncology, Emory University, Atlanta, GA, USA; ${ }^{2}$ Department of Radiation Oncology, Amsterdam University Medical \\ Centers, Amsterdam, The Netherlands \\ Correspondence to: Dr. Kristin A. Higgins. Department of Radiation Oncology, Emory University, 1365 Clifton RD NE, Atlanta, GA 30322, USA. \\ Email: Kristin.higgins@emory.edu.
}

Submitted Feb 18, 2020. Accepted for publication Mar 25, 2020.

doi: $10.21037 /$ jtd.2020.03.15

View this article at: http://dx.doi.org/10.21037/jtd.2020.03.15

Recent advances in the systemic treatment for extensive stage small cell lung cancer (ES-SCLC) have led to chemoimmunotherapy as part of the new standard of care. Both the IMpower 133 (1) and CASPIAN (2) trials demonstrated an improvement in overall survival (OS) with the addition of immunotherapy to first-line chemotherapy. Notably these trials did not allow definitive consolidative thoracic radiation, given limited data regarding safety of thoracic radiation and immunotherapy. As a result, there is currently uncertainty around if and how to integrate consolidative thoracic radiation into the chemo-immunotherapy backbone for ES-SCLC. This editorial commentary will address these timely and important issues.

The lack of progress in small cell lung cancer therapeutics has been halted with the recent IMPower 133 and CASPIAN trials, both of which demonstrated an OS benefit with the addition of immune check point inhibitors to first line platinum/etoposide chemotherapy in untreated, extensive stage small cell lung cancer. Prior to these studies, few breakthroughs had been made over the past several decades (3). The most notable advances involved radiotherapy, with PCI showing a survival benefit in ES-SCLC patients who responded to systemic therapy (4), as well as those receiving consolidative thoracic radiotherapy (5). It is noted that the PCI benefit may be less in patients who undergo routine three month surveillance with magnetic resonance imaging, as demonstrated by a Japanese study (6).

In the CREST trial, 30 Gy thoracic radiation was delivered after platinum/etoposide chemotherapy in patients that had any response to chemotherapy and did not have pleural or brain metastases. Patients receiving thoracic radiation had a 2 -year OS survival benefit $(13 \%$ vs. $3 \%, \mathrm{P}=0.004$ and a 6 -month progression-free survival (PFS) benefit (24\% vs. 7\%, $\mathrm{P}=0.001$ ). However, prior to the chemo-immunotherapy era in ES-SCLC, thoracic radiation was not been uniformly adopted across the world. This may be due in part to the CREST trial demonstrating a 2-year OS benefit but not a 1-year OS benefit, which was the primary endpoint of the study. In the CREST trial, presence of intrathoracic disease after chemotherapy was a stratification factor and in a subsequent analysis, a significant difference in OS was seen in the 434 patients (out of a total of 495) who had residual disease after chemotherapy [33\% vs. $26 \%$ at 1 year, $12 \%$ vs. $3 \%$ at 2 years, $\mathrm{P}=0.044$ (7)]. Irrespective of the survival benefit, consolidative thoracic radiation to $30 \mathrm{~Gy}$ in patients who have responded to systemic therapy is useful for prolongation of OS and PFS. Another possible reason for inconsistent use of consolidative thoracic radiation is conflicting data, particularly a study by the Radiation Therapy Oncology Group, RTOG 0937, which did not demonstrate a survival benefit to consolidative radiation to the chest combined with irradiation of distant metastatic sites (8).

Now in the era of chemoimmunotherapy for ESSCLC, should further evaluation of thoracic radiation be considered? It should be noted that the treatment effect of immunotherapy in both the IMpower 133 and CASPIAN trials was modest, with median OS improved by $2-3$ months in the experimental arms for both trials. There are no biomarkers that predict response to immunotherapy 
currently for ES-SCLC, and it is unclear which patients will benefit most from immunotherapy. Further research is underway to understand if immunotherapy combinations will further improve efficacy in ES-SCLC patients. Given consolidative thoracic radiation alone has shown improvements in OS after chemotherapy, and preclinical and growing clinical data has shown synergy of immunotherapy with radiation, it is rationale to further explore thoracic radiation in this context.

The hypothesis of radiotherapy/immunotherapy synergy is based upon radiation effects on both tumor cells and the host immune system. In preclinical models, adding concurrent checkpoint inhibitors to radiation was synergistic in xenograft models of pancreatic, colon, and breast cancer $(9,10)$. Both chemotherapy and radiation affect immune response to cancer by decreasing T-regulatory cells and by increasing expression of hidden tumor neoantigens during immune reconstitution after treatment. Radiation also leads to tumor-antigen release and a tumor specific adaptive immune response, which is enhanced by immune-stimulating agents. Thus, while radiation induces a local tumor response at the site of radiation, it may also lead to regression of micrometastatic disease (the abscopal effect) (11).

Dovedi et al. reported that low doses of fractionated radiation given concurrently with anti $\mathrm{PD}-1$ antibodies enhanced local tumor control and elicited out of field responses in a mouse model of colon cancer. Next generation sequencing of $\mathrm{T}$ cell receptors showed that while radiation increased $\mathrm{T}$ cell infiltration at the irradiated tumor only, radiation + anti PD-1 increased T cell infiltration/ expansion at both irradiated and out-of-field tumors. Additionally, radiation + anti-PD-1 increased T-cell receptor diversity more than radiation or anti PD-1 alone (12). Thus it is rationale that adding thoracic radiation could potentially lead to further improvements in PFS and OS in ES-SCLC treated with chemo-immunotherapy, to a greater degree than what has been observed in the prior CREST trial.

Extrapolating from the metastatic non-small cell setting, radiation in combination with immunotherapy has led to some provocative findings. In a study by Shaverdian et al., patients on Keynote 001 treated at a single institution were retrospectively analyzed for receipt of radiotherapy, and patients who had received radiotherapy had significantly improved PFS (median 4.4 vs. 2.1 months, $\mathrm{P}=0.019$ ) and $\mathrm{OS}$ [median 10.7 vs. 5.3 months, $\mathrm{P}=0.026$, (13)]. These findings support preclinical work showing synergy of radiation and immune check point inhibitors and support the underlying rationale of combining radiation to improve clinical outcomes in patients receiving immunotherapy.

Important safety data for the combination of immunotherapy and thoracic radiation in ES-SCLC has recently been published. A phase 1 study of 38 patients from MD Anderson showed thoracic radiation and concurrent pembrolizumab to be well tolerated, with no grade $4 / 5$ toxicities, and only $6 \%$ experienced a grade 3 toxicity (14). These data support further investigation of thoracic radiation and immunotherapy as treatment strategy in the chemo-immunotherapy era.

Additionally, the combination of nivolumab plus ipilimumab combined with thoracic radiotherapy (30 Gy in 10 fractions) following standard platinum-based chemotherapy was evaluated in a Phase I/II study, that was planned to enroll 52 ED-SCLC patients over two parts. The first part of the study was planned to establish the recommended dose of immunotherapy when combined with thoracic radiotherapy for the phase II study, whereas Part II was planned to estimate PFS. Results were presented at ASTRO 2019; the study closed after the first 21 patients were enrolled due to a planned interim analysis. The authors reported immune related adverse events (IRAEs) possibly or definitely attributable to study therapy, with a rate of $19.1 \%$ grade 3 or higher pulmonary IRAEs. Six month PFS estimate was $24 \%$, which was not significantly improved compared with historic controls, hence early study closure. However, the toxicity profile of immunotherapy and thoracic RT was felt to be consistent with what would be typically attributed to nivolumab and ipilimumab alone (15).

NRG Oncology LU007 is a phase II/III trial to evaluate the consolidative thoracic radiation to the chest and extra-thoracic sites after 4-6 cycles of carboplatinum/etoposide/atezolizumab. In this trial, patients will be randomized to radiation (up to 5 sites maximum) + atezolizumab $v s$. maintenance atezolizumab only. Stratification factors include number of isocenters ( $3 v s .3$ or more) performance status, and stable $v s$. responding disease. PCI will be optional and at the investigator's discretion. This trial will enroll 300 patients and will activate in 2020. This trial will answer an important question regarding the utility of both thoracic radiation and treatment of distant metastatic sites in the chemo-immunotherapy era.

\section{Acknowledgments}

Funding: None.

\section{Footnote}

Provenance and Peer review: This article was commissioned 
by the Guest Editor (Jose M. Pacheco) for the series "Small Cell Lung Cancer", published in Fournal of Thoracic Disease. This article did not undergo external peer review.

Conflicts of Interest: Both authors have completed the ICMJE uniform disclosure form (available at http://dx.doi. org/10.21037/jtd.2020.03.15). The series "Small Cell Lung Cancer" was commissioned by the editorial office without any funding or sponsorship. KAH reports other from Astra Zeneca, grants from RefleXion Medical, other from Varian, during the conduct of the study. BJS reports grants and personal fees from Varian medical systems, grants and personal fees from ViewRay Inc., outside the submitted work.

Ethical Statement: The authors are accountable for all aspects of the work in ensuring that questions related to the accuracy or integrity of any part of the work are appropriately investigated and resolved.

Open Access Statement: This is an Open Access article distributed in accordance with the Creative Commons Attribution-NonCommercial-NoDerivs 4.0 International License (CC BY-NC-ND 4.0), which permits the noncommercial replication and distribution of the article with the strict proviso that no changes or edits are made and the original work is properly cited (including links to both the formal publication through the relevant DOI and the license). See: https://creativecommons.org/licenses/by-nc-nd/4.0/.

\section{References}

1. Horn L, Mansfield AS, Szczesna A, et al. First-Line Atezolizumab plus Chemotherapy in Extensive-Stage Small-Cell Lung Cancer. N Engl J Med 2018;379:2220-9.

2. Paz-Ares L, Dvorkin M, Chen Y, et al. Durvalumab plus platinum-etoposide versus platinum-etoposide in firstline treatment of extensive-stage small-cell lung cancer (CASPIAN): a randomised, controlled, open-label, phase 3 trial. Lancet 2019;394:1929-39.

3. Gazdar AF, Bunn PA, Minna JD. Small-cell lung cancer: what we know, what we need to know and the path forward. Nat Rev Cancer 2017;17:725-37.

4. Slotman B, Faivre-Finn C, Kramer G, et al. Prophylactic cranial irradiation in extensive small-cell lung cancer. $\mathrm{N}$ Engl J Med 2007;357:664-72.

5. Slotman BJ, van Tinteren H, Praag JO, et al. Use of thoracic radiotherapy for extensive stage small-cell lung cancer: a phase 3 randomised controlled trial. Lancet 2015;385:36-42.

6. Takahashi T, Yamanaka T, Seto T, et al. Prophylactic cranial irradiation versus observation in patients with extensivedisease small-cell lung cancer: a multicentre, randomised, open-label, phase 3 trial. Lancet Oncol 2017;18:663-71.

7. Slotman BJ, Faivre-Finn C, van Tinteren H, et al. Which patients with ES-SCLC are most likely to benefit from more aggressive radiotherapy: A secondary analysis of the Phase III CREST trial. Lung Cancer 2017;108:150-3.

8. Gore EM, Hu C, Sun AY, et al. Randomized Phase II Study Comparing Prophylactic Cranial Irradiation Alone to Prophylactic Cranial Irradiation and Consolidative Extracranial Irradiation for Extensive-Disease Small Cell Lung Cancer (ED SCLC): NRG Oncology RTOG 0937. J Thorac Oncol 2017;12:1561-70.

9. Blanquicett C, Saif MW, Buchsbaum DJ, et al. Antitumor efficacy of capecitabine and celecoxib in irradiated and lead-shielded, contralateral human BxPC-3 pancreatic cancer xenografts: clinical implications of abscopal effects. Clin Cancer Res 2005;11:8773-81.

10. Deng L, Liang H, Burnette B, et al. Radiation and antiPD-L1 antibody combinatorial therapy induces T cellmediated depletion of myeloid-derived suppressor cells and tumor regression. Oncoimmunology 2014;3:e28499.

11. Tang $\mathrm{C}$, Wang $\mathrm{X}$, Soh $\mathrm{H}$, et al. Combining radiation and immunotherapy: a new systemic therapy for solid tumors? Cancer Immunol Res 2014;2:831-8.

12. Dovedi SJ, Cheadle EJ, Popple AL, et al. Fractionated Radiation Therapy Stimulates Antitumor Immunity Mediated by Both Resident and Infiltrating Polyclonal T-cell Populations when Combined with PD-1 Blockade. Clin Cancer Res 2017;23:5514-26.

13. Shaverdian N, Lisberg AE, Bornazyan K, et al. Previous radiotherapy and the clinical activity and toxicity of pembrolizumab in the treatment of non-small-cell lung cancer: a secondary analysis of the KEYNOTE-001 phase 1 trial. Lancet Oncol 2017;18:895-903.

14. Welsh JW, Heymach JV, Chen D, et al. Phase I Trial of Pembrolizumab and Radiation Therapy after Induction Chemotherapy for Extensive-Stage Small Cell Lung Cancer. J Thorac Oncol 2020;15:266-73.

15. Perez BA, Kim S, Dilling TJ, et al. A prospective Single Arm Phase I/II Study: Consolidative Ipilimumab and Nivolumab with Thoracic Radiotherapy after Platinum Based Chemotherapy for Patients with Extensive Stage Small Cell Lung Cancer. Int J Radiat Oncol Biol Phys 2019;105:S36.

Cite this article as: Higgins KA, Slotman BJ. What is the role of consolidative thoracic radiotherapy in the era of chemoimmunotherapy for extensive stage small cell lung cancer? J Thorac Dis 2020;12(10):6308-6310. doi: 10.21037/jtd.2020.03.15 